\title{
Clinical and laboratory versus molecular markers for a correct classification of von Willebrand disease
}

\author{
Augusto B Federici and Maria T. Canciani \\ Department Angelo Bianchi Bonomi Hemophilia and Thrombosis Center, Department of Internal Medicine and Medical \\ Specialities, IRCCS Foundation Maggiore Policlinico Hospital, Mangiagalli Regina Elena and University of Milan. \\ E-mail: augusto.federici@unimi.it.doi:10.3324/haematol.2009.005751
}

$\mathrm{V}$ n Willebrand disease (VWD) is the most common inherited bleeding disorder and is due to quantitative or qualitative defects of von Willebrand factor (VWF). VWD is inherited by autosomal dominant or recessive patterns, but women with mild forms are more symptomatic. ${ }^{1-3}$ In populationbased studies, the prevalence of VWD is very high $(0.81 \%),{ }^{4}$ but the clinical relevance of many of these cases is uncertain. Considering patients referred for clinical manifestations of bleeding, the actual prevalence is 66-100 cases per million of the general population. ${ }^{1-3}$ The most updated classification of VWD has proposed six different types: VWD1, VWD3, VWD2A, VWD2B, VWD2M, VWD2N. ${ }^{5}$ A partial quantitative defect marks VWD1, whereas VWD3 is characterized by the nearly total absence of VWF in plasma and platelets. VWD2A and VWD2B are marked by the absence of high molecular weight VWF multimers in plasma but in VWD2B there is also an increased affinity of VWF for its platelet receptor, the glycoprotein Ib alpha $(\mathrm{GpIb} \alpha)$. The identification of qualitatively abnormal variants with decreased platelet-dependent function and a normal multimeric structure marks VWD2M. VWD2N shows a full array of multimers, the defect being in the $\mathrm{N}$-terminal region of the VWF where the binding domain for FVIII is located. This type is phenotypically distinguishable from mild hemophilia A only by the abnormal binding of FVIII to VWF (VWF:FVIIIB). Correct classification of different types by clinical and laboratory parameters is important for management of patients with VWD. ${ }^{5}$

\section{Clinical and laboratory diagnosis}

Three main criteria are required for correct diagnoses of VWD: a) positive bleeding history since childhood; b) reduced VWF activity in plasma; c) history of bleeding in the family with autosomal dominant or recessive inheritance. The clinical and laboratory versus molecular markers useful for VWD diagnosis and classification are listed in Table 1: the dynamic use of these markers is shown in a flow-chart (Figure 1). The clinical markers include both personal and family history of bleeding: the presence of other affected members within the family is important to evaluate the autosomal dominant or recessive inheritance. Due to the multifunctional activities of VWF, more than one laboratory test is needed for VWD diagnosis. The ristocetin cofactor activity (VWF:RCo) is the most useful test for diagnosis, because it can mimic the interactions between VWF and platelets. Since the VWF is the carrier of factor VIII (FVIII), patients with very low levels of VWF have also relatively low concentrations $(<20 \mathrm{U} / \mathrm{dL})$ of FVIII. The diagnosis of mild VWD forms, particularly VWD1, may require several laboratory tests to be repeated on different occasions. Evidence-based diagnosis of VWD1 has been recently proposed according to these three criteria as published. ${ }^{6}$

\section{Clinical markers}

Clinical manifestations are excessive mucocutaneous bleeding and prolonged oozing after surgical procedures. In women, menorrhagia may be the only clinical manifestation. Soft tissue and joint bleeding are rare, except in patients with VWD3, characterized by severe deficiencies of VWF and FVIII. The clinical expression of the disease is usually mild in most patients with VWD1 and VWD2N, whereas severity increases in VWD2M, VWD2B, VWD2A and particularly in VWD3. Generally, the severity of bleeding correlates with the degree of reduction of VWF:RCo and FVIII. Several attempts have been recently made to evaluate sensitivity and specificity of bleeding symptoms, especially in the mild cases with VWD1 characterized by VWF:RCo levels $>20 \mathrm{U} / \mathrm{dL}$. In a multicenter study carried out in obligatory carriers of VWD1, menorrhagia and epistaxis were poor predictors of the disease while cutaneous bleeding and bleeding after dental extractions were more sensitive symptoms for diagnosis. ${ }^{7}$ A bleeding severity score (BSS) has been analyzed in affected and non-affected members of 154 families enrolled prospectively in a large European study on VWD1. Despite the fact that this BSS was investigated prospectively in VWD1, this approach can be useful in all VWD, as recently proposed in another prospective study. ${ }^{9}$ The bleeding time (BT), the original hallmark of the disease, is not always prolonged and may be normal in patients with mild forms, such as those with VWD1 and VWD2N. ${ }^{1-3}$ Hence, it is not particularly useful for diagnosis. Evaluation of closure time (CT) with the Platelet Function Analyzer (PFA-100) gives rapid and simple measurement of VWF dependent platelet function at high shear stress: it can be performed in whole blood and can therefore be employed instead of the BT in children or when the BT is not feasible. This system is sensitive and reproducible for VWD screening, but the CT is normal in VWD2N and cannot be modified in VWD3 after the administration of VWF/FVIII concentrates. ${ }^{10}$ Based on these observations, BT and CT are not introduced in the flow-chart to be used in the differential diagnosis of VWD types (Figure 1).

\section{Laboratory markers}

Unlike hemophilia A, the diagnosis of which only requires two parameters, namely the prolonged partial thromboplastin time (PTT) with low levels of FVIII, several laboratory tests should be performed to diagnose VWD types (Table 1). Among these tests to be 
performed sequentially as first and second levels (Figure 1), the ristocetin cofactor activity (VWF:RCo) explores the interaction of VWF with the platelet GPIb $\alpha$ and is still the standard method for measuring VWF activity. VWF:RCo is based on the property of the antibiotic ristocetin to agglutinate formalin-fixed normal platelets in the presence of VWF. This method is specific for VWF abnormalities but it is not very sensitive (values $<6 \mathrm{U} / \mathrm{dL}$ not reliable) and not always reproducible (CV inter- and intra assay 8-15\%). Among several other methods developed more recently, the novel ELISA VWF:RCo assays using recombinant or plasma-derived GPIb offers increased (as low as $<1$ U/dL) sensitivity and lower (5-8\%) CV. ${ }^{11}$ VWF antigen (VWF:Ag) is a very sensitive assay when it is measured by ELISA test: VWF:Ag is undetectable in VWD3, is reduced in VWD1 and can be normal in most VWD2A, VWD2B, VWD2M, VWD2N. In patients with a normal VWF structure (VWD1 and VWD2N), VWF:RCo values are similar to VWF:Ag (VWF:RCo/Ag ratio >0.7); VWF:RCo/Ag ratio $<0.7$ are characteristic of VWD2A, VWD2M and of most cases with VWD2B, as reported in the guidelines for diagnosis and treatment of VWD in Italy. ${ }^{3}$ In the past VWD1 was reported to be the most frequent form of VWD, accounting for approximately $70 \%$ of cases. A reappraisal of VWD diagnoses after ten years (1998-2008) in 1,234 Italian patients showed only $671 / 1,234(55 \%)$ patients with VWD1, because many cases previously diagnosed as VWD1 were rediagnosed VWD2A or VWD2M due to discrepant

Table 1. Clinical and laboratory versus molecular markers used for VWD diagnosis.

i) Clinical markers for VW/

- Clinical history: lifelong mucocutaneous and post-operative bleeding, to be collected with appropriate questionnaires to calculate the Bleeding Severity Score (BSS)

- Family history positive for bleeding and/or other affected VWD

T) Laboratory markers for correct dlagnosis of WWD types

First Level:

- VWF: Ristocetin cofactor activity [a]

- VWF antigen [b]

- Factor VIII [c]

- Ristocetin Induced Platelet Agglutination (RIPA) [d]

- VWF:RCo/Ag [e] and FVIII/WWF:Ag [f]

Second Level:

- VWF multimeric structure on low and high resolution gels [g]

- VWF pro-peptide $[h]$ measured as ratio with VWF antigen

(VWFpp/WF:Ag)

- Infusion test [i] with desmopressin (DDAVP)

- Factor VIII binding assay [I]

\section{i) Molecular markers for dlagnosis confirmation of WWD}

Search for large deletion in VWD3

Search for mutations clustered within specific VWF domains:

D2-D3-C2-A2-CK (VWD2A); D3 (VWD1/2M Vicenza) D'-D3 (VWD2N); $\mathrm{Al}$ (VWD2B and VWD2M)

For the use of these tests see the diagnostic flow-chart reported in Figure 1.
VWF:RCo/Ag ratio. ${ }^{9}$ The presence of qualitative defects of VWF in previously diagnosed VWD1 has also been reported in 154 families evaluated prospectively by the European Study. ${ }^{12}$ In this European cohort of patients the VWF:RCo/Ag ratio $<0.6$ was predictive of structural abnormalities and of mutations within specific regions of VWF gene: ${ }^{12}$ therefore we have introduced the cut-off level of 0.6 for the VWF:RCo/Ag ratio in this updated flow-chart (Figure 1). The procoagulant activity of factor VIII (FVIII) is usually very low (1-5 U/dL) in patients with VWD3 who are characterized by undetectable levels of VWF:Ag. In patients with VWD2A, VWD2B, VWD2M, FVIII is normal in most cases. VWF is the carrier of FVIII and therefore in normal individuals both proteins can be found in equal amounts in circulation as FVIII/VWF complex with FVIII/VWF ratio equal to 1 . The FVIII/VWF:Ag ratio can be a useful laboratory marker, because such a ratio is $>1$ in VWD1 and $<1$ in VWD2N..$^{1-3}$

Ristocetin-induced platelet agglutination (RIPA) is measured by mixing in the aggregometer different concentrations of ristocetin and patient platelet rich-plasma (PRP). Results are expressed as the concentrations of ristocetin $(\mathrm{mg} / \mathrm{mL})$ able to induce $30 \%$ agglutination. Most VWD types show a low response to ristocetin $(>1.2 \mathrm{mg} / \mathrm{mL}$ of ristocetin concentration), but an important exception is VWD2B, in which there is hyper-responsiveness to ristocetin $(<0.8 \mathrm{mg} / \mathrm{mL})$, due to a higher than normal affinity of VWF for platelet GPIb $\alpha .{ }^{13}$ Normal VWF is composed of a complex series of multimers with molecular weight ranging from 800 to 20,000 kiloDaltons $(\mathrm{kDa})$, which can be analyzed by agarose gel electrophoresis. Low-resolution agarose gels distinguish VWF multimers, which are conventionally indicated as high, intermediate and low molecular weight. In VWD1, VWD2M and VWD2N all multimers are present, whereas in VWD2A the high and intermediate multimers are missing. Most VWD2B show the loss of high multimers even though there are patients with relatively normal multimers. ${ }^{14}$ VWF multimeric analysis with high-resolution agarose gels can be useful to further characterize patients with VWD2A (VWD2A, subtypes IIC, IID, IIE, IIF, IIG, IIH), as reported. . $^{1-3,5}$

The VWF propeptide (VWFpp) and VWF proteins remain non-covalently associated and stored in alphagranules in megakaryocytes/platelets or Weibel-Palade bodies in endothelial cells for regulated release. In plasma, VWFpp and mature multimers dissociate and circulate independently. VWFpp circulates in plasma as a homodimer with a half-life of 2-3 hours, while mature VWF circulates with a half-life of 8-12 hours. ${ }^{5}$ For these reasons, the ratio between VWFpp and VWF:Ag has been recently proposed to identify VWD1 patients with reduced VWF survival. ${ }^{15}$

Desmopressin (1-deamino-8-D-arginine vasopressin, DDAVP) is a synthetic analog of vasopressin that it is relatively inexpensive and carries no risk of transmitting blood-borne infectious agents. DDAVP, infused intravenously at a dose of $0.3 \mu \mathrm{g} / \mathrm{Kg}$ diluted in $50 \mathrm{ml}$ saline over 30 minutes, usually increases plasma VWF and FVIII 3-5 times above baseline levels within 30-60 
minutes and, in general, high VWF and FVIII levels last for 6-8 hours. ${ }^{1-3}$ A test dose of DDAVP is recommended in VWD patients at the time of diagnosis to establish the individual patterns of biological response and to predict clinical efficacy during bleeding, since the responses in a given patient are consistent on different occasions. ${ }^{3}$ Such a DDAVP infusion trial should be considered an important tool for VWD diagnosis, because VWD patients can be divided according to their biological response in three different groups: short halflife, responsive, not responsive. ${ }^{16,17}$ Note that an increased ratio of VWFpp/VWF:Ag usually correlates with short half-life of VWF activities after DDAVP ${ }^{15}$ and that partial or low biological response of DDAVP can be predicted by reduced levels or abnormal structure of platelet VWF. ${ }^{1-3}$ Knowledge of the biological response after such an infusion trial at the time of diagnosis is really important because VWD patients can be immediately identified as unresponsive or short-life responsive to DDAVP and should be shifted immedi- ately to the use of plasma-derived VWF/FVIII concentrates. $^{1-3}$

The VWF binding assay to FVIII (VWF:FVIIIB) measures the affinity of VWF for FVIII. In this assay, antiVWF antibody is coated on wells of a micro titer plate and test plasma is added to the wells. The FVIII/VWF complex from the plasma is bound by the antibody after which FVIII is removed from the complex by a high ionic strength buffer. Excess recombinant FVIII (rFVIII) is then added and, after removal of unbound rFVIII, the VWF and the bound rFVIII are assayed..$^{1-3,5}$ This assay allows VWD2N to be distinguished from mild to moderate hemophilia A.

\section{The role of molecular diagnosis}

Molecular markers

Molecular and prenatal diagnoses of VWD have been introduced since the early 1990s. Originally, the first mutations were found within exon 28 of the VWF gene that is responsible for domains A1, A2, A3. Most

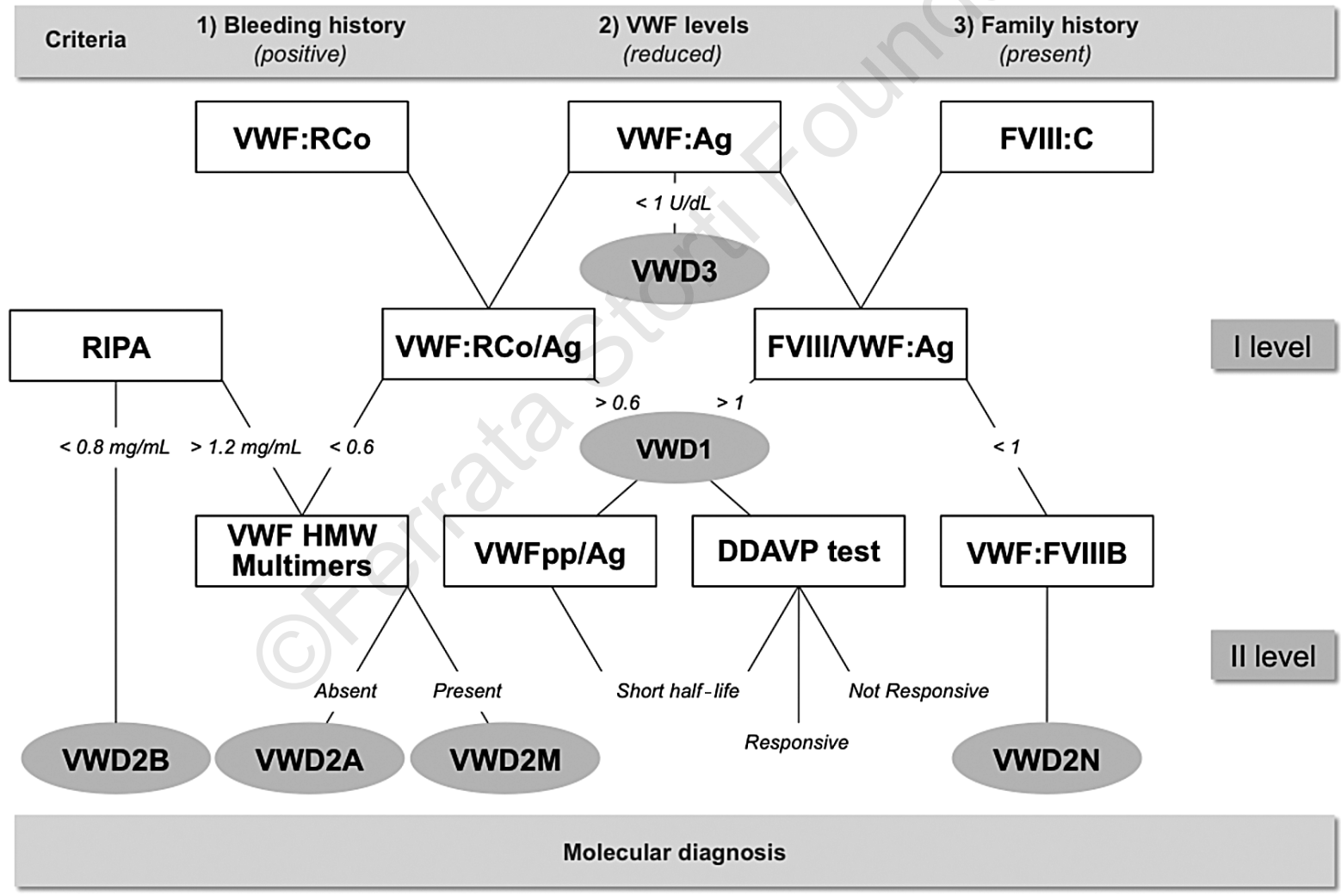

Figure 1. Flow-chart proposed for the correct diagnosis and classification of different VWD types. Once bleeding history of the suspected patients with VWD is collected and family history of bleeding investigated (Table 1), a reduced level of VWF should be measured by using VWF:RCo (a). First level of diagnosis: VWD3 can be diagnosed in case of undetectable VWF:Ag (b). FVIII (c) is always reduced in VWD3 and in VWD2N: it can be reduced or normal in all the other VWD types. VWD2B can be identified (d) in case of heightened RIPA ( $<0.8 \mathrm{mg} / \mathrm{mL}$ ) whereas VWD1, VWD2A and VWD2M cause low RIPA ( $1.2 \mathrm{mg} / \mathrm{mL}$ ). A proportionate reduction of both VWF:Ag and VWF:RCo with a VWF:RCo/Ag ratio > 0.6 suggests VWD1 (e). If the VWF:RCo/Ag ratio is < 0.6, VWD2A, VWD2B, and VWD2N should be suspected. VWD2N can be suspected in case of FVIII/VWF ratio $(f)<1$ while a FVIII/VWF ratio is $>1$ is usually associated with VWD1. Second level of diagnosis: Multimeric analysis in plasma (g) is necessary to distinguish between VWD2A (lack of the largest and intermediate multimers) and VWD2M (all the multimers present). VWFpp/VWF:Ag $(h)$ is increased in VWD1 with short half-life of VWF. DDAVP infusion test (i) can identify patients with no biological response, short biological response or response to this drug. VWF:FVIIIB (i) should be performed to confirm VWD2N. This figure is modified and updated from that reported previously. ${ }^{3}$ Once phenotypic diagnosis is performed, mutations should be searched for (molecular diagnosis) to confirm VWF defects within the family of VWD patients (Figure 2). 
VWD2A cases are due to missense mutations in the A2 domain, with R1597W or Q or Y and S1506L accounting for about $60 \%$ of them. The majority of VWD2B cases are due to missense mutations in the A1 domain, about $90 \%$ being caused by R1306W, R1308C, V1316M and R1341O. ${ }^{1-3,5,14}$ A few heterogeneous mutations, also located within the A1 domain, underlie VWD2M. A recurrent mutation in VWD1/2M Vicenza has been identified in families from Europe (R1205H), another mutation (M740I) is seen exclusively in families from the Vicenza area in the North East of Italy. ${ }^{18,19}$ Missense mutations in the FVIII-binding domain at the amino-terminal portion of VWF are responsible for VWD2N. ${ }^{20}$ The molecular defects responsible for VWD2A, VWD2B, VWD2M and VWD2N are located in specific VWF domains (Figure 2): on the other hand, mutations responsible for VWD1 and VWD3 are spread within the entire VWF gene.

The genetic causes of VWD1 are still elusive in many cases, especially in those with a mild phenotype and VWF levels above $30 \mathrm{U} / \mathrm{dL}$. More information on the molecular basis of VWD1 has been collected by two multicenter international studies. In the European study, recruitment was based on the historical diagnosis of VWD1, which included 278 affected cases and
312 non-affected family members. ${ }^{12}$ The Canadian investigators could recruit 123 families for which the index case had bleeding symptoms and VWF levels between 5 and $50 \mathrm{U} / \mathrm{dL} .{ }^{21}$ In this study, subjects with abnormal multimeric patterns or other evidence of qualitative defects were excluded. The most important conclusions from both studies are the following: a) despite the selection of patients based on bleeding history, candidate VWF mutations were not found for $27 \%$ (Canadian) and 36\% (European) of index cases diagnosed with VWD1; b) the spectrum of VWD1 mutations was different from that found in VWD3. Therefore, VWD1 is not at all like heterozygous VWD3 because VWF defects that occur in VWD1 are usually caused by dominant VWF abnormalities that affect VWF secretion or clearance without altering multimeric patterns or platelet binding.

In VWD3, partial or total gene deletions have been initially reported..$^{22}$ Notably, homozygosity for gene deletion may be associated with the appearance of allo-antibodies against VWF, which may render replacement therapy ineffective and stimulate anaphylactic reactions to treatment. ${ }^{23,24}$ In general, mutations may be scattered over the entire gene, but some (e. g. 2680delC or Arg2535X) are particularly recurrent in Northern Europe. ${ }^{25}$ Gene defects of VWD3 patients
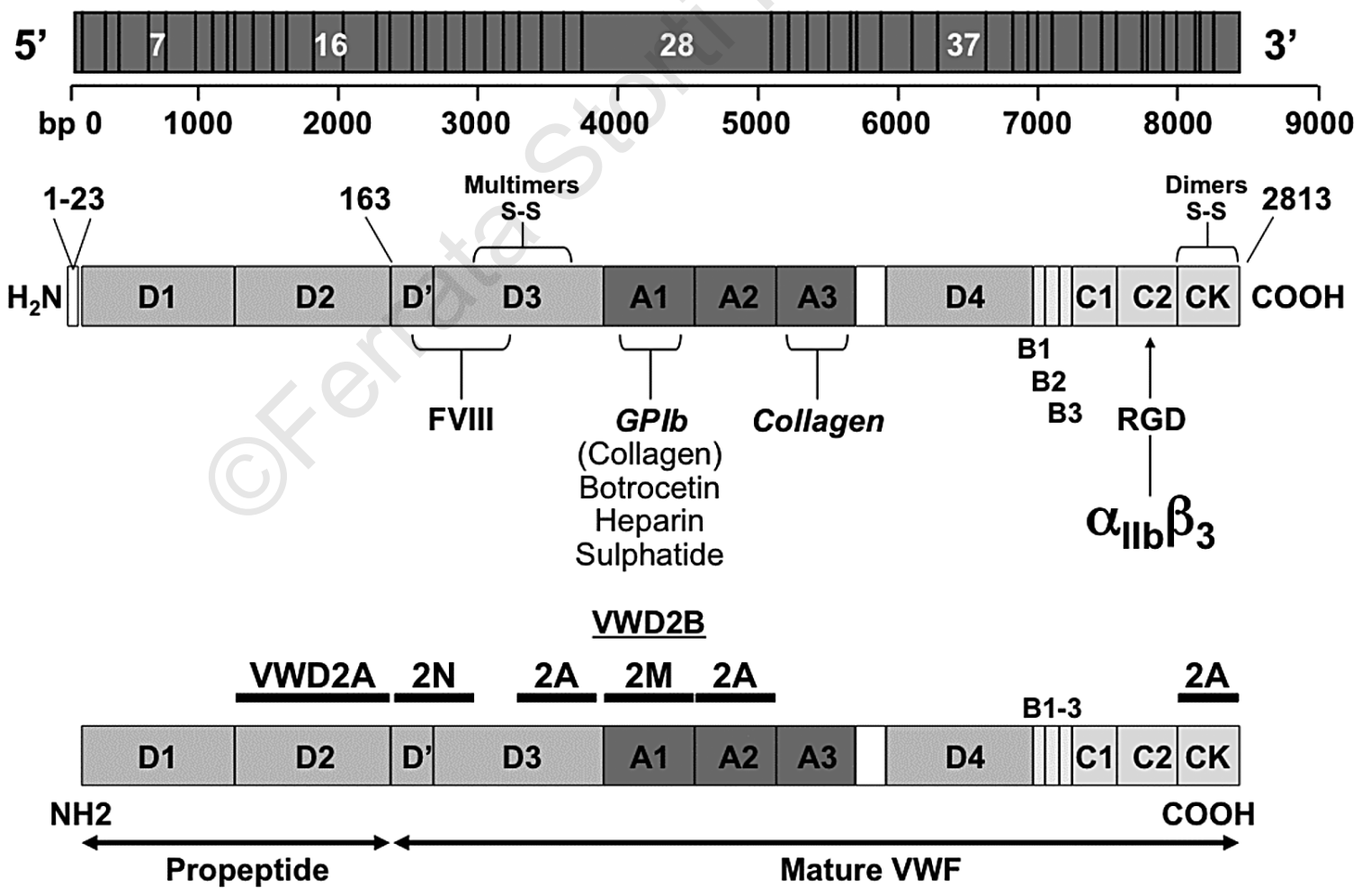

Figure 2. Schematic representation of the VWF gene located in chromosome 12: the main exons are indicated with the number of base pairs from 5' to 3' (upper panel). The structure of VWF functional domains: the pre-pro-VWF is indicated with amino acids numbered from the amino- (aa 1) to carboxy-terminal portions (aa 2813) of VWF. Note the important CK and D3 domains for formation of VWF dimers and multimers. The native mature subunit of VWF, after the cleaving of the pre-pro-VWF, is described with its functional domains: the VWF binding sites for factor VIII (D' and D3), Gplb, botrocetin, heparin, sulfatide, collagen (A1), collagen (A3) and the RGD sequence for binding to -llb,3 (intermediate panel). Distribution of VWF mutations in patients with VWD2: the positions of mutations causing VWD2A, VWD2B, VWD2M, VWD2N are indicated with black bars throughout the VWF domains (lower panel). 
from three different populations have now been studied, but there was no founder effect and mutations were distributed throughout the entire VWF gene. ${ }^{26}$ Compared to hemophilia, most VWD patients show relatively mild bleeding symptoms. Therefore, prenatal diagnosis is required mainly in cases of parents already known to be carriers of VWD3, with gene defects identified in their first affected child. ${ }^{27}$ Since young children with VWD3 might carry deletions of VWF gene that predispose to the allo-antibodies to VWF, every new child with VWD3 should be intensively investigated by searching for deletions before starting extensive therapy with exogenous VWF concentrates.

A good example of how this diagnostic approach with clinical, laboratory and molecular markers can be useful to better characterise patients with VWD has been provided by a report by Pérez-Rodriguez et al. ${ }^{28}$ in this issue of the Journal. These authors have further investigated three unrelated families characterized by mutation C1149R that was previously associated with VWD1 diagnosis. By using second level laboratory and DDAVP tests (Figure 1), they could demonstrate that the VWF structure and response to DDAVP were not consistent with VWD1 and therefore they proposed that this group of patients should be classified as VWD2A, subtype IIE. This report suggests that an appropriate laboratory test (second level) and a clinical trial with DDAVP should be always performed to confirm classification of VWD type even though mutations have already been found.

In summary, diagnosis of VWD can benefit from better clinical, laboratory and molecular markers. The contribution of clinical and laboratory data has been recently evaluated by a Bayes theorem approach and evidence-based diagnosis of VWD1 has been proposed. ${ }^{6}$ Following this experience, the use of the bleeding severity score quantifying the bleeding history of the patients should be introduced in all VWD patients to evaluate the potential risk of bleeding of each VWD case, as recently proposed in another prospective study. ${ }^{9}$ The phenotypic diagnosis is still the most accessible on account of the difficulties and costs of molecular diagnosis. The use of more sensitive and precise VWF:RCo assays should improve the laboratory diagnosis of VWD, especially when levels of VWF are $<15$ $\mathrm{U} / \mathrm{dL}$. The DDAVP infusion trial should always be introduced during the diagnostic flow of any new patient with VWD, because it can provide additional information on the VWF activities released from endothelial cells and identify cases who can benefit from this treatment during bleeding episodes or surgery. Molecular diagnosis can be useful to confirm specific VWF defects in VWD families, especially those with VWD2A, VWD2B, VWD2M and VWD2N since mutations are clustered in specific exons of VWF gene. In VWD3 patients, no specific mutations can be used as molecular markers for the disease, since gene defects are spread within the entire VWF gene; however, large deletions should be searched for because they can be associated with the appearance of allo-antibodies against VWF. In VWD1, the probability of finding mutations within the entire VWF gene is high only when VWF levels are below $30 \mathrm{U} / \mathrm{dL}$. It is still not clear whether most mild VWD1 patients really have a mutation in the VWF locus and the possibility of external modifiers of VWF levels should be considered. Despite its complex and heterogeneous nature, nowadays patients can be efficiently diagnosed and classified in most Western Countries. A correct diagnosis and classification of VWD is always important to provide the best therapeutic approach to these patients.

Dr. Federici is an Associate Professor of Hematology at the University of Milan, while Dr. Canciani works at the Angelo Bianchi Bonomi Hemophilia Thrombosis Center, Milan, Italy.

\section{References}

1. Castaman G, Federici AB, Rodeghiero F, Mannucci PM. Von Willebrand's disease in the year 2003: towards the complete identification of gene defects for correct diagnosis and treatment. Haematologica 2003;88:94-108.

2. Federici AB, Mannucci PM. Management of inherited von Willebrand disease in 2007. Ann Med 2007;39:346-58

3. Federici AB, Castaman G, Mannucci PM. Guidelines for the diagnosis and management of VWD in Italy. Haemophilia 2002;8:607-21.

4. Rodeghiero F, Castaman G, Dini E. Epidemiological investigation of the prevalence of von Willebrand's disease. Blood 1987;69:454-9.

5. Sadler JE, Budde U, Eikenboom JC, Favaloro EJ, Hill FG, Holmberg L, et al.; Working Party on von Willebrand Disease Classification. Update on the pathophysiology and classification of von Willebrand disease: a report of the Subcommittee on von Willebrand Factor. J Thromb Haemost 2006;4:2103-14

6. Tosetto A, Castaman G, Rodeghiero F. Evidence-based diagnosis of type 1 von Willebrand disease: a Bayes theorem approach. Blood 2008;111:3998-4003.

7. Rodeghiero F, Castaman G, Tosetto A, Batlle J, Baudo F, Cappelletti A, et al. The discriminant power of bleeding history for the diagnosis of type 1 von Willebrand disease: an international, multicenter study. J Thromb Hemost 2005;3: 2619-26.

8. Tosetto A, Rodeghiero F, Castaman G, Goodeve A, Federici AB, Batlle J, et al. A quantitative analysis of bleeding symptoms in type 1 von Willebrand disease: results from a multicenter European study (MCMDM-1 VWD). J Thromb Haemost 2006;4:766-73.

9. Federici AB, Bucciarelli P. Castaman G, Mazzucconi MG Morfini M, Schiavoni M, Mannucci PM. Prevalence and Determinants of bleeding in different types of von Willebrand disease: results of the first prospective multicenter study on 814 Italian patients. Blood 2007, Abstract 713.

10. Cattaneo M, Federici AB, Lecchi A, Agati B, Lombardi R, Stabile F, Bucciarelli P. Evaluation of the PFA-100 system in the diagnosis and therapeutic monitoring of patients with von Willebrand disease. Thromb Haemost 1999;82:35-9.

11. Federici AB, Canciani MT, Forza I, Mannucci PM, Marchese P, Ware J, Ruggeri ZM. A sensitive ristocetin cofactor activity assay with recombinant glycoprotein Ibalpha for the diagnosis of patients with low von Willebrand factor levels. Haematologica 2004;89:77-85.

12. Goodeve A, Eikenboom J, Castaman G, Rodeghiero F, Federici AB, Batlle J, et al. Phenotype and genotype of a cohort of families historically diagnosed with type 1 von Willebrand disease in the European study, Molecular and Clinical Markers for the Diagnosis and Management of Type 1 von Willebrand Disease (MCMDM-1VWD). Blood 2007;109:112-21.

13. Ruggeri ZM, Pareti FI, Mannucci PM, Ciaverella N, Zimmerman TS. Heightened interaction between platelets and factor VIII/von Willebrand factor in a new subtype of von Willebrand disease. $N$ Engl J Med 1980;302:1047-51.

14. Federici AB, Mannucci PM, Castaman G, Baronciani L, 
Bucciarelli P, Canciani MT, et al. Clinical and molecular predictors of thrombocytopenia and risk of bleeding in patients with von Willebrand disease type 2B: A cohort study of 67 patients. Blood 2009;113:526-34.

15. Haberichter SL, Castaman G, Budde U, Peake I, Goodeve A, Rodeghiero F, et al. Identification of type 1 von Willebrand disease patients with reduced von Willebrand factor survival by assay of the VWF propeptide in the European study: molecular and clinical markers for the diagnosis and management of type 1 VWD (MCMDM1VWD). Blood 2008;111:4979-85.

16. Federici AB, Mazurier C, Berntorp E, Lee CA, Scharrer I, Goudemand J, et al. Biologic response to desmopressin in patients with severe type 1 and type 2 von Willebrand disease: results of a multicenter European study. Blood 2004;103:2032-8

17. Castaman G, Lethagen S, Federici AB, Tosetto A, Goodeve A, Budde U, et al. Response to desmopressin is influenced by the genotype and phenotype in type 1 von Willebrand disease (VWD): results from the European Study MCMDM-1VWD. Blood 2008;111:3531-9.

18. Schneppenheim R, Federici AB, Budde U, Castaman G, Drewke E, Krey S, et al. Von Willebrand Disease type 2M "Vicenza" in Italian and German patients: identification of the first candidate mutation (G3864A; R1205H) in 8 families. Thromb Haemost 2000;83:136-40.

19. Castaman G, Missiaglia E, Federici AB, Schneppenheim $\mathrm{R}$, Rodeghiero F. An additional unique candidate mutation (G2470A; M740I) in the original families with von Willebrand disease type $2 \mathrm{M}$ Vicenza and the G3864A (R1205H) mutation. Thromb Haemost 2000;84:350-1.

20. Mazurier C, Goudemand J, Hilbert L, Caron C, Fressinaud E, Meyer D. Type $2 \mathrm{~N}$ von Willebrand disease: clinical manifestations, pathophysiology, laboratory diagnosis and molecular biology. Best Pract Res Clin Haematol 2001;14: 337-47.
21. James PD, Notley C, Hegadorn C, Leggo J, Tuttle A, Tinlin S, et al. The mutational spectrum of type 1 von Willebrand disease: results from a Canadian cohort study. Blood 2007; 109:145-54

22. Shelton-Inloes BB, Chebab FF, Mannucci PM, Federici AB, Sadler JE. Gene deletions correlate with the development of alloantibodies in von Willebrand disease. J Clin Invest 1987;79:1459-65.

23. Mannucci PM, Tamaro G, Narchi G, Candotti G, Federici A, Altieri D, Tedesco F. Life-threatening reaction to factor VIII concentrate in a patient with severe von Willebrand disease and alloantibodies to von Willebrand factor. Eur J Haematol 1987;39:467-70.

24. Mannucci PM, Federici AB. Antibodies to von Willebrand factor in von Willebrand disease. Adv Exp Med Biol 1995; 386:87-92.

25. Eikenboom JC. Congenital von Willebrand disease type 3: clinical manifestations, pathophysiology and molecular biology. Best Pract Res Clin Haematol 2001;14:365-79.

26. Baronciani L, Cozzi G, Canciani MT, Peyvandi F, Srivastava A, Federici AB, Mannucci PM. Molecular characterization of a multiethnic group of 21 patients with type 3 von Willebrand disease. Thromb Haemost 2000;84:536-40.

27. Peake IR, Bowen D, Bignell P, Liddell MB, Sadler JE, Standen G, Bloom AL. Family studies and prenatal diagnosis in severe von Willebrand's disease by polymerase chain reaction amplification of a variable number of tandem repeat region of the von Willebrand factor gene. Blood 1990;76:555-61.

28. Pérez-Rodríguez A, García-Rivero A, Lourés E, LópezFernández MF, Rodríguez-Trillo A, Batlle J. Autosomal dominant C1149R von Willebrand disease: phenotypic findings and their implications. Haematologica 2009; 94:679-86.

\section{Factor IX and deep vein thrombosis}

\section{Gordon Lowe}

University of Glasgow, Royal Infirmary, Glasgow, UK. E-mail: g.d.lowe@clinmed.gla.ac.uk

doi:10.3324/haematol.2009.005769

T hrombosis has been described as hemostasis in the wrong place. ${ }^{1}$ Fibrin is a major component of thrombi and anticoagulant drugs which reduce thrombin formation are effective in both prevention and treatment. So, increased circulating levels of coagulation factors, and their functional genotypes, appear prime candidates for mechanisms of both arterial and venous thrombosis. Recent reviews of the epidemiological literature have concluded that testing of these hypotheses, while showing some promising results, still has a long way to go. ${ }^{2,3}$

At present, increased levels of the von Willebrand factor: factor VIII complex show the most consistent associations with both venous and arterial thrombosis, in both case-control and prospective studies..$^{2-6}$ For the moment, the causality of these associations cannot be tested, because there are no drug or other interventions which selectively reduce levels of von Willebrand factor (VWF) or factor VIII. However, Mendelian randomization studies support their causal roles in venous and arterial thrombosis. Non O-blood group is associated with increased risk of both venous and arterial thrombosis ${ }^{7}$ which is most likely due to mean $25-30 \%$ higher circulating levels of VWF: factor VIII complex, due to the expression of non-O proteo- glycans on VWF which reduce its clearance from the circulation by the reticulo-endothelial system. Hemophiliacs, who have low levels of Factor VIII (or IX), have a low risk of arterial thrombosis such as coronary heart disease (CHD). ${ }^{8}$ A study of CHD risk in hemophilia carriers in the Netherlands ${ }^{8}$ suggested that this lower risk is dose-dependent, being intermediate in hemophilia carriers, who have on average about half the circulating levels of Factor VIII (or IX) compared to non-carrier females.

These reports suggest that levels of factors VIII and IX (which have critical roles in hemostasis, as shown by the severe clinical bleeding manifestations of hemophilias A and B) may also play important roles in venous and arterial thrombosis. While factor VIII is an important cofactor in hemostasis, factor IXa is the activated enzyme which plays a key role in maintenance of thrombin (and hence fibrin) formation within the intrinsic system of coagulation. Clinical, epidemiological, and pharmacological studies support the hypothesis that increased factor IX levels play a role in thrombogenesis. ${ }^{9}$

There are two approaches to test this hypothesis. First, development of anticoagulant drugs which selectively reduce factor IX levels, ${ }^{9}$ and randomized trials of 